\title{
LE DISCOURS SYNDICAL SUR LA FORMATION ET LE RÔLE DES ENSEIGNANTS AU QUÉBEC, 1930-1990
}

\author{
M'hammed Mellouki \\ Patricia-Anne Côté \\ Monique L'Hostie
}

Si le principal but de toute association professionnelle est de promouvoir les interêts des membres, la forme que prend son organisation, la nature même, la portée et les fondements sur lesquels repose son engagement, de même que les cadres juridiques qui délimitent son champ d'action sont en revanche largement tributaires des forces sociales en présence, des conjonctures économiques, politiques et idéologiques que traverse la société dans son ensemble. Parmi les associations formées et transformées par les enseignants du secteur catholique du système scolaire de la province du Québec au cours des soixante dernières années, la Corporation générale des instituteurs et des institutrices catholiques (CIC) - fondée en 1946 mais dont les origines remontent aux années 1930n'échappe pas à cette règle. La CIC-qui devient Corporation des enseignants du Québec, en 1967, puis Centrale de l'enseignement du Québec (CEQ), en 1974--, représente en effet le plus grand regroupement de syndicats d'enseignants et l'organisation qui a joué un rôle de première importance dans la structuration de la profession d'enseigner, dans la promotion des interêts des enseignants, de leur rôle et statut et dans l'amélioration de leurs conditions de travail. Les revendications qu'elle formulera, les luttes qu'elle mènera et les alliances qu'elle conclura n'auront, tout au long des années 1930-1990, qu'un seul et même but: démontrer l'importance et l'utilité sociale de la mission que remplissent les éducateurs et, par voie de conséquence, la nécessité de la valorisa. tion de l'enseignant, de son rôle et de son statut social. Les orientations ideologiques et les moyens d'action de l'association changent radicalement au gré des conjonctures économiques, politiques et idéologiques, mais son but demeure le même. C'est là le fil conducteur de l'êtude qui fait l'objet du présent article.

L'analyse du discours de la CEQ est susceptible de renseigner sur l'évolution de cette importante catégorie professionnelle que forment les enseignants, sur le 
rôle et le statut des membres, sur l'emprise qu'ils ont ou celle qu'ils tentent d'établir sur leur propre travail et sur les institutions sociales et politiques. En ce sens le discours de la CEQ représente non seulement un espace où on doit suivre, à travers le temps, le déploiement des actions de l'organisation visant la définition et la légitimation de la place de l'enseignant dans l'école et dans la société, mais aussi un lieu où on peut déceler certaines transformations que subissent, sous l'impact des conjonctures et des divisons internes, ses orientations idéologiques et ses rapports à la société et à l'État.

Nous tenterons dans le présent article de suivre le mouvement interne du discours syndical pour en dégager les thèmes dominants et, quand cela s'avère possible, compte tenu de l'espace dont nous disposons, de repérer la logique qui le lie ou oppose aux appareils iđéologico-politiques: l'Église et l'État notamment. C'est dans ce cadre d'analyse compréhensif et socio-historique - dont nous avons délimité les contours dans des travaux antérieurs - que s'insère donc l'étude consacrée ici au discours syndical. Toutefois, même si l'approche adoptée epouse intentionnellement, dans le but justement de la devoiler, la perspective des acteurs concernés-les élites syndicales-, nous essayerons, à chaque fois que cela nous paraîtra utile et surtout dans la conclusion, de mettre en évidence le jeu de certains déterminants externes et de tensions et divisions internes qui ont contribué à la structuration du discours de la CEQ sur l'école et sur les enseignants, leur formation, leur rôle et leur profession.

Force enfin est de constater que, exception faite de la thèse de Marta Anadon ${ }^{2}$, le discours syndical n'a jamais vraiment fait l'objet d'analyse sociohistorique et compréhensive. Aussi le corpus documentaire est-il constitué ici essentiellement de textes de première main, de sources imprimées surtout, même

1. Voir M'hammed Mellouki, «Les agents scolaires, leur place et fonction dans les rapports sociaux: le cas des inspecteurs d'écoles (1851-1964) et des conseillers d'orientation (1930-1990) au Québec», Sociétés contemporaines, 1992 a (à paraître); «Rhétorique universitaire el savoir enseignant», dans M'hammed Mellouki, Maurice Tardif et Clermont Gauthier, Les savoirs des enseignants, leur formation el les fondements de leur compétence professionnelle. Montréal, Editions Logiques, 1992b; à paraitre, «La qualification des enseignants: un enjeu et ses acteurs», dans Claude Lessard, Madeleine Perron et Pierre-W. Bélanger, La profession enseignante au Québec: enjeux et défis pour les années 1990, Québec, IQRC, 1991a, p. 43-67; «Les personnels de l'enseignement du Québec, 1930-1990», Revue des sciences de l'éducation, vol. XVII, no 3, 1991b, 365-387; «Évolution des programmes de formation des enseignants au Québec de 1930 à 1960: un cas de rupture idéologique», Revue d' histoire de l'éducation, printemps 1990a, 37-58; «Les discours sur le savoir enseignant au Québec, quelques repères socio-historiques, 1930-1989», Revue des sciences de l'éducation, vol. XVI, no.3, automne 1990b, 393-404; Savoir et idéologie réformiste, la formation des mâtres, 1930-1964 (Québec, IQRC, 1989), 293-303.

2. Marta Anadon, L'école québécoise: jeux el enjeux de forces sociales, 1970.1980 (Québec, Laboratoire de recherches sociologiques, Université Laval, 1989). 
si des travaux sur le syndicalisme enseignant sont rappelés ça et là pour éclairer le contexte du discours et aider à saisir les transformations qui s'y opèrent.

Ceci étant dit, trois moments sont distingués dans l'évolution du discours syndical. Le premier couvre la periode allant des années 1930 à la grande réforme scolaire des années 1960. Il s'agit là d'une période importante dans la structuration de la profession. Le deuxième désigne la période qui s'étend de la réforme scolaire à la fin des années 1970, soit entre le moment où la CIC entame, suite à des événements extraordinaires, la réorientation de son idéologie et de son action qui va la conduire tout droit vers la radicalisation de son engagement socio-politique et vers une confrontation ouverte avec l'État, et le moment où elle commence à modérer son radicalisme. Enfin, le troisième moment couvre, grosso modo, la période des années 1980 où la CEQ, en même temps qu'elle tente d'ajuster son idéologie et ses actions en fonction des caractéristiques de la conjoncture politique et économique, consacre l'essentiel de ses efforts à préserver son unité et à conserver ses acquis. La CEQ semble ici délaisser le discours radical au profit d'une adhésion à l'idéologie du pluralisme idéologique à laquelle, cependant, elle ne s'était jamais directement opposée.

\section{STRUCTURATION DE LA PROFESSION}

Les efforts et les actions visant l'organisation de la profession furent menés sur les deux principaux fronts habituellement presents dans toute lutte pour la reconnaissance sociale d'un corps de métier: le premier étant celui de la mise sur pied d'associations qui ont pour but la défense des intérêts de ceux et celles qui exercent le dit métier et le second celui de la définition, de l'amélioration et surtout de la recherche du contrôle des critères de reconnaissance des savoirs et des savoir-faire propres à ce champ d'activite. Évidemment, les actions se déplacèrent de l'un à l'autre front selon les besoins et les circonstances qui se présentaient, selon le degré de conscience qu'avaient les membres de leur force et de la communauté de leurs intérêts, selon les rapports qu'ils entretenaient avec les appareils politiques et idéologiques.

\section{Syndicalisme et corporatisme enseignant}

Les luttes pour l'organisation de la profession ne datent pas des années trente $^{3}$. Mais c'est au cours de cette décennie, et sous l'impulsion vigoureuse

3. Voir à ce sujet André Labarrère-Paulé, Les laiques et la presse pédagogique au Canada français au XIXe siècle (Québec, Les presses de l'Université Laval, 1963); 
d'une institutrice, Laure Gaudreault, que les premiers syndicats d'institutrices rurales s'incorporent (1936) en vertu de la Loi des syndicats professionnels de 1924. La fin des années 1930 est marquee par de nombreuses luttes, visant une amélioration des conditions de travail et des salaires amputés pendant la grande crise économique, et par la création des Fédérations regroupant le personnel enseignant laïque des écoles primaires publiques du territoire provincial.

L'annee 1946 marque un pas important dans l'histoire de l'organisation de la profession enseignante au Québec. En décembre 1945, la Fédération catholique des institutrices rurales de la province de Québec, celle des instituteurs ruraux ainsi que la Fédération des instituteurs et institutrices des cités et des villes, se regroupent et fondent la Corporation generale des instituteurs et institutrices catholiques de la province de Québec (CIC). Le 17 avril 1946, le gouvernement Duplessis vote la loi corporative reconnaissant la $\mathrm{CIC}$. Cette loi tisse un fort lien de dépendance entre l'État et le syndicalisme enseignant. À l'origine, la Corporation souhaitait rassembler les läques légalement qualifiés pour enseigner aux secteurs privé et public, peu importe leur religion et leur langue. Elle doit se contenter de regrouper les laïques des écoles primaires publiques catholiques selon les limites imposées par le gouvernement Duplessis. Ce dernier refuse de lui accorder le pouvoir de contrôler l'admission à la pratique. Cette loi imprègne donc le syndicalisme enseignant d'un caractère ambigu, mi-corporatiste, mi-syndical, en marge du mouvement ouvrier organisé. Toutefois, même si elle n'est pas maitresse de sa constitution, la CIC peut exercer un pouvoir réglementaire et disciplinaire, imposer une contribution à ses membres et négocier en leur nom des conventions collectives.

À cette époque, le personnel enseignant est composé en grande majorité de femmes. Le métier exige peu de qualifications, les salaires sont bas et le pouvoir des commissions scolaires dans l'embauche et le congédiement du personnel enseignant devient à peu près total surtout après l'adoption par le gouvernement Duplessis, en 1946, d'une loi supprimant le droit à l'arbitrage pour le personnel enseignant des municipalitếs rurales ${ }^{4}$. Ce droit ne sera rétabli qu'en 1959 sous le gouvernement de Paul Sauvé qui, en même temps, réintroduira et étendra à l'ensemble des enseignants de l'élémentaire et du secondaire des écoles publiques catholiques la clause de l'adhésion obligatoire à la ClC abolie en 1953. En obtenant du gouvernement ces amendements à sa loi constitutive, la $\mathrm{ClC}$ verra ses effectifs passer de 16000 a 28000 membres en l'espace d'un an ${ }^{5}$.

Les instituteurs laiques au Canada français, 1836-1900 (Québec, Les presses de l'Université Laval, 1965); "L'instituteur läque canadien français au $19 \mathrm{e}$ siècle», dans Marcel Lajeunesse, L'éducation au Québec 19e-20e siècles (Montréal, Boréal Express, 1971), 59.76.

4. Province du Québec, Loi pour assurer le progrès de l'éducation (Québec, province de Québec, 1946).

5. Jacques Rouillard, Histoire du syndicalisme au Québec, des origines à nos jours 
De la fin des années 1930 à la fin des années 1950 , les actions des syndicats d'enseignants se concentrent sur l'amélioration du régime de pension, des conditions de travail et de rémunération des membres, ainsi que sur la promotion de la «profession». La CIC revendique pour ses membres un salaire qui assure leur subsistance, les incite à persévérer dans la profession et leur garantit une certaine sécurité pour l'avenir. De telles améliorations rendraient plus attrayante la profession aux candidats compétents et contribueraient à la résolution de l'épineux problème du fort taux de roulement du personnel enseignant en lui assurant la stabilité et les avantages qui conviennent «à l'importance de ses fonctions» ${ }^{6}$. Les syndicats ont contribué pendant toutes ces années à informer leurs membres sur des sujets à caractère non seulement syndical tels la doctrine sociale de l'Église, le contrat collectif ou le perfectionnement, mais aussi sur des sujets de nature pédagogique comme l'organisation de la classe, les qualités de l'institutrice ou les devoirs à domicile.

Ceux et celles qui ont travaillé à la création de la CIC rêvaient d'une véritable corporation professionnelle qui contrôle l'admission à la pratique et jouit d'un pouvoir réglementaire et disciplinaire. La loi corporative de 1946 en décida autrement. Durant les deux décennies qui ont suivi, ce «rêve» ainsi que le système de valeurs qui lui est sous-jacent sont restés bien vivants au coeur des discours, des politiques et des revendications de la Corporation. Ainsi, le congrès de 1957 allait à nouveau réclamer «la profession fermée (...) afin que la CIC soit légalement et pleinement constituée comme autorité au service de la profession»?. En fait, la $\mathrm{ClC}$ ne renoncera à ce désir de contrôler l'admission à la pratique, qu'aurait dû lui permettre le statut de corporation fermée, qu'à la fin des années soixante sous le poids de la conjoncture politique et des nouveaux rapports de force qui s'établissent entre les équipes dirigeantes ${ }^{8}$.

\section{Une profession en quête de reconnaissance}

Dès sa création en 1946, la CIC se donne pour mission de faire reconnaître le statut professionnel de ses membres. Elle recommande à ces derniers de refuser l'exécution des travaux de ménage et d'entretien des classes, travaux qu'elle considère incompatibles avec la dignité de la profession'. Elle tentera, au cours des années suivantes, par le biais de diverses publications, brochures, dépliants et annonces publicitaires dans les médias, de mieux faire connâtre la

(Montréal, Boréal, 1989), 23.

6. ClC, Voeux des congrès annuels 1953-1965 (Québec, CIC, 1965), section C, 1.

7. Ibid, 3 .

8. Voir à ce sujet M'hammed Mellouki, «La qualification des enseignants, un enjeu et ses acteurs», loc. cil, 1991a.

9. Ibid, sect.C, 2 . 
profession d'enseignant et demandera à ses membres «d'intéresser leurs meilleurs élèves à la carrière» et d'éveiller, « dès maintenant, l'intérêt des jeunes pour l'éducation» ${ }^{10}$. Pour valoriser le statut et le rôle social de ceux et celles qui oeuvrent dans le champ de l'enseignement, la Corporation suggère à tous ses membres dûment qualifiés d'inscrire I.D., après leur nom, pour: Instituteur et Institutrice Diplômés. Elle demande également aux fédérations et aux synđicats affiliés de multiplier, chacun dans sa région, les publications, les réunions et toute initiative permettant aux enseignants d'assumer un rôle de promoteurs de la culture. En 1959, le IX ${ }^{\mathbb{e}}$ congrès recommande au conseil d'administration de la Corporation d'organiser une campagne de promotion «pour mieux faire comprendre au peuple le caractère hautement professionnel et spécialisé de la carrière de l'enseignement» ${ }^{11}$ et de mettre sur pied un bureau de publicité qui utilisera à cette fin les médias. Cinq ans plus tard, soit en 1964, le XIV congrès conclue que le public méconnaissait toujours «la tâche des instituteurs» et qu'il fallait une autre campagne d'information pour «rehausser le prestige de la profession ${ }^{12}$. L'appel lancé à la société, en 1961, afin qu'elle prenne «toutes les mesures requises pour donner aux instituteurs et institutrices de la province de Québec un véritable statut professionnel» ${ }^{13}$, était resté, semble-t-il, lettre morte.

Parce qu'elle represente les «professionnels» de l'enseignement, la Corporation ne cesse donc, depuis sa création et, surtout, entre 1953 et 1965, de réclamer le droit de siéger dans divers comités et sous-comités du Département de l'Instruction publique et d'être représentée au sein du Comité catholique du Conseil de l'Instruction publique. Par exemple, elle exige en 1954, 1961 et 1962 de siéger au comité de régie des examens officiels. Elle estime que les instituteurs et les institutrices doivent être consultés parce qu'ils sont les experts et les praticiens de l'enseignement. Le IX ${ }^{\mathfrak{c}}$ congrès de 1959 déplore «les situations regrettables engendrées par le fait que les instituteurs et institutrices n'aient aucune voix au chapitre de la vie scolaire» ${ }^{14}$, alors même qu'ils ont le «désir» et le «droit» de participer activement aux processus de prise de décision.

Pour conquérir le prestige depuis longtemps désiré, la Corporation incite également ses membres à se comporter comme des professionnels dans leur milieu social. C'est pourquoi elle se réjouit, en 1957, de la participation croissante de ses membres à des émissions culturelles sur les ondes de Radio-Canada. C'est pourquoi elle encourage les sections diocésaines à oeuvrer pour «cultiver l'honneur professionnel» ${ }^{15}$ chez leurs membres. En plus de posseder le «sens

\footnotetext{
10. CIC, op. cit, 1965, section C, 3 .

11. Ibid, 5 .

12. Ibid, p. 11, c'est nous qui soulignons.

13. Ibid, 5 .

14. Ibid, 4.

15. Ibid, voeux du congrès de 1957, 5 .
} 
professionnel», l'éducateur doit d'être competent dans son domaine et cultive ${ }^{16}$. L'éducateur est aussi un citoyen responsable qui a, plus que tout autre, développé, de par la formation qu'il a acquise et les fonctions qu'il exerce, «une vision aiguë des lacunes et besoins de la vie civique et politique de son milieu» ${ }^{17}$. Enfin, toujours dans le but de hausser la qualification de ses membres et la valeur sociale et économique de la profession enseignante, la Corporation demande, dès 1958 , au Département de l'Instruction publique la suppression du brevet $\left\langle\mathrm{C} \gg{ }^{18}\right.$. Elle considere qu'une seule anné d'études dans une école normale ne suffit pas à préparer adéquatement une personne à l'enseignement, compte tenu de l'évolution rapide que connaissent dans les dernières décennies les techniques pédagogiques et de la complexité de plus en plus grande des problèmes d'éducation. Un statut professionnel exige de son détenteur la possession d'un haut degré de culture génerale et des qualifications professionnelles nécessitant au moins deux années de préparation, autant pour les institutrices que pour les instituteurs ${ }^{19}$. Si les écoles secondaires s'acquittent convenablement de leur mission d'institutions de formation fondamentale et si les normes d'admission aux écoles normales sont haussées, les contenus des programmes révisés et la durée des études pédagogiques est augmentee, la qualification du personnel enseignant en sortira améliorée et le statut du métier, revalorisé ${ }^{20}$. C'est dans ce sens que, des 1957, la CIC considere, par exemple, que toutes les écoles normales devraient dispenser des cours de méthodologie de l'enseignement audio visuel, cours qui, si l'on juge par les expériences tentées dans d'autres pays, contribuent à l'amelioration du rendement scolaire des elèves ${ }^{21}$. En 1962, elle revient à la charge et demande d'accélérer la formation des mâtres pour l'enseignement secondaire et d'augmenter le temps alloué à l'enseignement pratique dans les programmes de formation. Aux yeux de la Corporation, l'elévation des normes imposées aux normaliens et aux normaliennes à l'admission et en cours de formation devrait contribuer à redorer le blason de la profession.

Par ailleurs, le contexte politique et scolaire de la fin des années 1940 et de toute la décennie 1950 fut marqué d'evénements importants qui n'ont pas manqué de revigorer l'action syndicale et le discours qui le supportait. Sur le plan politique, on peut rappeler à titre d'exemple la grève historique menée par les professeurs de l'Alliance de Montréal en 1949, l'adoption, à la suite de cette

16. Ibid, voeux du congrès de 1961, 6 .

17. Ibid, 6 .

18. Le brevet «C» a été aboli en 1956, deux ans seulement après sa création, des écoles normales d'hommes. Il ne sera abrogé des écoles normales de femmes qu'en 1962. Voir à ce sujet M'hanmed Melloukj, Savoir enseignant et idéologie réformiste, la formation des mat̂tres, 1930-1964, op. cit., 1989, 216.

19. CIC, op. cil, 1965, voeux du congrès de 1961, 4-5.

20. Ibid, 6 .

21. Jbid, section F, 1 . 
grève, de la loi 60 posant de sérieuses limites au droit d'arbitrage dont jouissaient encore les enseignants des centres urbains, la promulgation en 1954 de la loi 20 , rétroactive à 1944, qui interdit le recours à la grève dans le secteur public, et le rétablissement en 1959 de l'obligation de l'adhésion de tous les enseignants catholiques, les religieux et religieuses exceptés, à la $\mathrm{ClC}$, obligation qui, en l'espace de deux ans, haussa de $143 \%$ l'effectif de l'organisation 22 .

Sur le plan scolaire, après l'adoption de la loi sur l'obligation scolaire en 1943 et la mise en vigueur d'une réforme substantielle des programmes scolaires en 1948, les années 1950, on s'en souvient, seront chargées d'événements à plus d'un égard décisifs pour l'avenir du métier d'enseigner: réforme importante qui donna, a partir de 1953, aux écoles normales le statut d'établissements de formation professionnelle et haussa la duree de la préparation des futurs instituteurs et institutrices; élargissement de l'accès à l'école secondaire publique, instaurée officiellement à partir de 1956, hausse continue du taux de natalité, explosion scolaire et crise de plus en plus aiguë de recrutement de nouveaux religieux et religieuses enseignants qui induisent un besoin urgent en instituteurs et institutrices laïques; institution d'une commission royale d'enquête sur les problèmes constitutionnels (1953-1956)-dont incidemment plus du tiers des mémoires qu'elle reçut portait sur l'éducation-qui consacra une importante étude $^{23}$ à la situation scolaire et conclut que, pour lui attirer des candidats talentueux, l'enseignement ne devait plus être consideré comme un apostolat mais reconnu comme une profession à part entière; sans oublier, enfin, la tenue en 1958 d'une conférence provinciale sur les problemes de l'éducation au Québec, la première du genre qui réunit les membres de l'intelligentsia canadienne-française, et dont la préparation a donné lieu à la création de l'Association d'Éducation du Québec (AÉQ) qui tiendra son premier congrès en 1960 sur la persévérance scolaire.

Si les traits de cette conjoncture ont donné un souffle d'énergie aux enseignants dans la revendication d'un statut mieux consideré et de meilleures conditions de travail, le discours de la CIC, quant à lui, est resté, on l'a vu, soumis aux limites idélogiques et politiques qui étaient celles que lui imposaient la doctrine sociale de l'Église et l'action législative de l'État, même si de jeunes militants commençaient ça et là à s'opposer aux dirigeants syndicaux en place ${ }^{24}$.

22. Voir Pierre Dionne, Une analyse historigue de la Corporation des Enseignants du Québec, 1836-1968, mémoire de mâtrise (relations industrielles), Université Laval, Québec, 1969, 162; voir aussi Bernard Dionne, Le syndicalisme au Québec, (Montréal, Boréal, 1991).

23. Tremblay, Arthur, Contribution à l'étude des problèmes et des besoins de l'enseignement dans la province de Québec (Québec, Gouvernement du Québec, 1955), 407 p.

24. Raymond Laliberté, Entrevue accordée à l'auteur le 6 avril 1990. 


\section{Le mémoire à la Commission Parent}

Dans le volumineux mémoire qu'elle présente à la Commission royale d'enquête sur l'enseignement (Commission Parent) en 1962, la CIC réserve une partie substantielle à l'analyse du statut professionnel du maître et de la formation initiale du personnel enseignant. En tentant de définir le statut professionnel du «maitre», la Corporation aborde la question du lien idéologique qui lui semble lier ce dernier à l'Église, à la famille et à l'État. En tant que membre d'une communauté confessionnelle, le «maître» doit reconnaitre, dans l'ordre, sa subordination à l'Église, à la famille et à la société civile ${ }^{25}$. Et puisque la première autorité est celle de l'Église, les éducateurs catholiques doivent s'opposer à un idéal d'éducation neutre qui «n'est possible qu'au coût d'une violation des droits de l'Église». Un tel idéal est «monstrueux» dans une «société à très forte majorité confessionnelle» ${ }^{26}$. Par ailleurs, en tant que «délégué de la famille», la première responsabilité du maittre est «de se conformer aux directives qui lui viennent de ses employeurs» 27 .

En tant qu'expert et professionnel, l'enseignant devra jouir d'une autonomie dans l'exercice de ses fonctions, avoir le droit đe négocier ses conditions de travail et d'être consulté sur toute question concernant son affectation. Aussi bien dans l'exercice de ses fonctions que dans les relations qu'il entretient avec ses supérieurs immédiats, le maître doit arborer «un esprit de liberté et d'auto-direction» ${ }^{28}$ et s'affranchir du «paternalisme» et de «l'autoritarisme» qui caractérisent traditionnellement ses rapports avec certains administrateurs scolaires àl'échelon local et provincial. Les superieurs immédiats, les commissaires, les inspecteurs et les directeurs d'écoles doivent plutôt apprendre à considérer le professionnel de l'enseignement comme un «collaborateur qualifié.» C'est d'ailleurs à ce titre que le Departement de l'Instruction publique devrait lui demander son avis à chaque fois qu'il est question des finalités et des moyens d'éducation, de la prolongation de la fréquentation scolaire obligatoire, de l'introduction de la télévision dans les écoles, de la réforme des programmes des écoles normales ou de toute autre question relative à l'enseignement et aux rapports entre les parents et les personnels de leur école.

Quels sont les critères qui devraient régir la formation de ces professionnels de l'enseignement que sont les enseignants? La ClC croit qu'il faudrait d'abord planifier et resserrer le contrôle de cette formation, éviter l'ouverture précipitée de centres de formation et le recrutement hâtif du personnel enseignant des écoles

25. CIC, Mémoire de la Corporation des instituteurs et institutrices catholiques du Québec à la Commission royale d'enquête sur l'enseignement (Québec, CIC, 1962), 39.

26. Ibid, 49 .

27. Ibid, 44 .

28. Ibid, 74 . 
normales, éliminer le corps des chargés de cours ou du moins en diminuer l'effectif et instaurer un mécanisme d'evaluation rigoureuse des besoins et des ressources disponibles ${ }^{29}$. La réforme scolaire devra, selon la Corporation, commencer par la révision du nombre et de la distribution des écoles normales sur le territoire québécois afin d'évaluer sérieusement leur rendement et de ne conserver que les meilleures d'entre elles. Les institutions maintenues n'engageront que des professeurs possédant des compétences reconnues dans leurs champs de spécialisation et ayant une expérience pertinente dans l'enseignement ${ }^{30}$. Ensuite, malgre les besoins pressants d'instituteurs et d'institutrices que provoque l'augmentation rapide de la clientele scolaire, le recrutement des normaliens ne devra pas continuer à se faire de façon précipitée, ni sur la base de critères approximatifs de sélection. Pour pallier à cette absence de rigueur dans la sélection des candidats, la $\mathrm{CIC}$ recommande à la commission Parent de mettre sur pied un organisme provincial «chargé d'établir aussi scientifiquement que possible les composantes de l'aptitude à l'enseignement ${ }^{31}$. Elle suggere également la création d'une classe préparatoire, dite année «Pré-Normale», qui constituera un «test de sélection» préliminaire des candidats à l'enseignement avant d'être acceptés en première année de formation pédagogique (ou première pédagogique). L'année «Pré-Normale» pernettra aux institutions de formation "d'étudier plus longuement les caracteristiques de qui voudra s'inscrire chez elles» et aux candidats de réfléchir et d'évaluer sérieusement leur choix de carrière. Enfin, cête sélection, de même que la nécessité d'une supervision psycho-pédagogique des normaliens, commandent la mise sur pied, dans chaque école normale aussi bien religieuse que laïque, d'un service de psychologues et de conseillers d'orientation.

Sur le plan des contenus de la formation, l'accent doit être mis sur les matières professionnelles davantage que sur les matières académiques ou de culture génerrale. Il faut toutefois reconnâtre qu'un nombre important de candidats admis dans les écoles normales ne possède pas une formation de base suffisante prealable. Cette lacune est souvent attribuée au faible rendement des écoles secondaires. C'est pour cette raison d'ailleurs que les cours de «méthodologie spéciale» (didactique), dispensés par les écoles normales, se transforment bien souvent en cours de transmission pure et simple de connaissances élémentaires en sciences naturelles, en français, en mathématiques ou en musique ${ }^{32}$. Aussi longtemps que la mâtrise des disciplines de base n'est pas assurée par l'école secondaire, l'ecole normale est obligée d'y remédier.

29. Ibid, 157 .

30. Jbid, 157.

31. Ibid, 161.

32. Ibid, 164. 
Dans le même ordre d'idées, la $\mathrm{CIC}$ juge insuffisante la durée réglementaire d'une semaine prévue annuellement pour l'initiation des normaliens à la pratique de l'enseignement. Et même là, certaines écoles les dispensent parfois de ce «strict minimum» ${ }^{33}$. La Corporation recommande que soit haussée à un minimum de trois semaines par année la durée du stage d'initiation a l'enseignement dans une école d'application ou dans toute autre institution approuvée aux mêmes fins.

Quant à la durée totale de la formation initiale, la CIC pense qu'elle devra $s " e ́ c h e l o n n e r$ sur un minimum de quatre années d'études effectuées après le diplôme de l'enseignement secondaire (11ème annee) $)^{34}$. La première année, dite annee pré-normale, sera consacrée à l'acquisition d'un complément de culture génerale. Les trois autres années seront réservées à la formation professionnelle proprement dite dont le contenu pourra être réparti de la manière suivante. La première année sera commune a tous. Elle favorisera une meilleure connaissance des programmes scolaires que les candidats auront à enseigner et l'acquisition d'une culture pédagogique. Les deux années subséquentes, sans négliger la formation theorique, devront faire une place importante à la pratique de l'enseignement, à l'étude de la législation scolaire et à la formation «syndicale». Le programme conduira à l'obtention d'un «brevet standard» d'enseignement. L'obtention d'un brevet unique mettra ainsi fin aux pratiques classificatoires utilisées par le Comité catholique du Conseil de l'Instruction publique, pratiques qui, conjuguees à la multiplication des nouveaux diplômes (sept ou huit brevets, titres ou certificats, en 1962) et à l'instabilité des programmes de formation (un programme n'est pas aussitôt proposé qu'on le révise), entraînent immanquablement la dévalorisation de certains brevets et la déqualification de leurs détenteurs. La CIC dénonce parfois avec humour de telles pratiques. Ainsi, écrit-elle, personne ne serait tenté de «confier la construction d'un édifice à un architecte de grade 2 , ni de faire dresser le cadastre d'une municipalite par un arpenteur de catégorie C». Comme ces professionnels, les enseignants doivent se doter d'une solide formation et obtenir un systeme de certification uniforme ${ }^{35}$.

La formation initiale devra d'abord conduire à un permis temporaire d'enseigner puis à un brevet permanent. Après avoir complété les études prévues, réssi les examens officiels et obtenu une recommandation favorable de son principal, le normalien se verra conférer par le surintendant de l'Instruction publique une «autorisation provisoire d'enseigner.» Celte autorisation accordera à son détenteur tous les droits généralement reconnus à un instituteur de métier. Après une période de «probation» d'une durée de «deux années d'enseignement avec succès», le permis deviendra un brevet permanent conférant un «plein statut

33. Ibid, 165.

34. Ibid, 144.

35. Ibid, 145 et 137. 
professionnel» à son détenteur ${ }^{36}$. Un tel système permettra de se prémunir contre les inconvénients éventuels d'une formation initiale souvent peu poussée, contre le risque d'admettre définitivement un candidat sans garanties sérieuses sur sa compétence à exercer une «activité fort complexe» ou un candidat insoucieux d'ameliorer sa qualification professionnelle. La réforme du système de certification est donc inévitable. Elle doit se faire «dans l'interêt du public autant que dans celui de la profession» ${ }^{37}$.

\section{ENSEIGNER ET CONSCIENTISER}

L'action syndicale menée par la CEQ au cours des années 1970 se présente, écrit Louise Clermont-Laliberté, comme «une suite de tentatives pour dépasser une certaine pratique réformiste au profit d'une pratique de transformation sociale ${ }^{38}$. C'est surtout suite a l'adoption de la loi 25 (1967), suspendant le droit de grève et de négociation et obligeant les enseignants en grève à retourner à leurs classes, que la CEQ commence à prendre ses distances vis-à-vis du ministère de l'Éducation avec qui elle a étroitement collaboré, de 1964 à 1966, à la mise en place de la reforme du système de formation des enseignants ${ }^{39}$. Au cours de cette même période elle abandonne sa loi corporative et se transforme en centrale syndicale: la Centrale de l'enseignement du Québec, «marquant par-là sa volonté d'indépendance vis-à-vis [de] l'État» ${ }^{40}$. En 1972, la CEQ participe à un Front commun avec la Confédération des syndicats nationaux (CSN) et la Fédération des travailleurs du Québec (FTQ) dans le but de renforcer leur position et d'obliger l'État à accélérer la négociation des conventions collectives des travailleurs du secteur public ${ }^{41}$. Entre 1972 et 1975 , elle publie trois manifestes dans lesquels elle va exposer sa nouvelle conception de la société, de l'école, de la pédagogie et du rôle de l'enseignant ${ }^{42}$. La CEQ prône désormais la pédagogie

\section{Ibid, 168 .}

37. Ibid, 167.

38. Louise Clermont-Laliberté, Dix ans de pratiques syndicales, CEQ 1970-1980, (Québec, CEQ, 1981), 67.

39. Voir à ce sujet $M$ 'hammed Mellouki, «La qualification des enseignants, un enjeu et ses acteurss, loc. cit, 1991a.

40. $\mathrm{CEQ}$, Formation et perfectionnement des enseignantes et enseignants du primairesecondaire, premier avis sur le document du MEQ (Québec, CEQ, janvie: 1984), 8.

41. CSN et CEQ, Histoire du mouvement ouvrier au Québec (s.l., coédition, CSN et CEQ, 1984), 265-269.

42. L'école au service de la classe dominante (Québec, CEQ, 1972); Ecole et luttes de classes au Québec (Québec, 1974); Manuel du ler mai (Québec, 1975). 
de conscientisation comme modèle devant être adopté par tous les enseignants. Au cours de la decennie 1970, elle se préoccupe en particulier de la question de la probation que doivent subir les nouvelles recrues avant d'obtenir leur permis permanent d'enseignants. Elle demande aux enseignants en place de defendre les intérêts des «probanistes» au sein des comités de probation cherchant ainsi $a$ réduire le pouvoir de l'État-patron sur cette question. Elle considere dorénavant la négociation collective comme «le lieu privilégié» de son intervention ${ }^{43}$. Aussi, même la question de la formation devient une question qui doit être débattue, au même titre que les autres, sur le terrain politique.

\section{Pour une co-direction du procès de la qualification des enseignants}

En 1967, trois ans après la création du ministère de l'Éducation et du Conseil supérieur de l'Éducation, la $\mathrm{CIC}$ devient la Corporation des enseignants du Quebec (CEQ) et publie l'année suivante un Manifeste sur la formation et le perfectionnement des enseignants. Ce document pose les prémices de la conception que défendrá au cours des années à venir la CEQ en matière de formation et de perfectionnement du personnel enseignant. Ce document dénote un changement dans le discours syndical. Désormais, deux acteurs sociaux doivent partager les responsabilités de la formation des futurs praticiens de I'enseignement: la CEQ et le MEQ. En 1968, plus question de subordination à l'Église et à la famille. La Corporation entend traiter d'égal à égal avec le ministère de I'Éducation. Le contrôle de la profession se fera sur une base birpartite: l'État faisant entendre «la voix de l'autorité publique» et la CEQ, celle des «spécialistes» de l'enseignement ${ }^{44}$. La co-direction aura pour objectifs: (a) d'estimer les besoins réels et le nombre de spécialistes requis en enseignement, (b) de déterminer les modalites de formation et (c) d'octroyer les permis d'enseignement et, surtout, de décider de la nature des critères d'octroi de ces permis ${ }^{45}$. Selon la CEQ, c'est aux spécialistes du domaine-les enseignants-de déterminer les critères de compétence et de contrôler la pratique de la profession, comme c'est le cas pour les médecins, les ingenieurs et les avocats ${ }^{46}$. Même s'il ne semble pas pressé de l'assumer autrement que par le biais de l'approbation des budgets, c'est au ministère de l'Éducation qu'incombe, selon la CEQ, la

43. $\mathrm{CEQ}$, La probation des enseignonts selon les règles administratives 22-10-08 pour 1979-80 VS les politiques de la CEQ (Québec, CEQ, 1979), 15.

44. CEQ, Manifeste de la Corporation des enseignants du Québec sur la formation et le perfectionnement des enseignants, (Québec, CEQ, 1968), Avant-propos el 26.

45. Ibid, 26-29.

46. Ibid, 28 . 
responsabilité de la coordination des programmes de formation dispensés par les universites ${ }^{47}$.

La formation des enseignants doit dorénavant s'acquérir dans des institutions de type universitaire, non parce que les écoles normales n'ont pas rempli adéquatement leur mission, ni parce que les universités sont bien préparées pour s'acquitter convenablement de cette tâche, mais plutôt pour répondre au besoin d'une plus grande «cohérence du système» et d'une revalorisation de la profession enseignante. Pour ce faire, les futurs enseignants sont appelés à quitter l'univers clos des écoles normales et à prendre le chemin des institutions où sont formés les autres professionnels ${ }^{48}$. Il est donc urgent, selon la CEQ, de creer l'Université du Québec et de réformer les universités existantes afin qu'elles puissent contribuer de manière appropriée et efficace a la préparation des éducateurs.

Leur nouvelle mission exige donc des universités une évaluation sérieuse de leurs pratiques pédagogiques dans le domaine de la formation du personnel enseignant. La CEQ formule dans ce manifeste une critique plutôt virulente de la pédagogie universitaire. Elle écrit: «Nous réclamons que la pédagogie soit stimulante pour l'etudiant et non paternaliste et constipante; nous exigeons des professeurs d'université un sérieux recyclage (...) en pédagogie (...) les travaux de Bloom sur la taxonomie des objectifs ou les perspectives ouvertes par Skinner ou Crowder en enseignement programmé ne peuvent plus être ignorés» 49 . Sur le plan plus spécifique de la formation en enseignement professionnel, la Corporation attend des universités qu'elles s'attachent plus particulierement à la préparation pédagogique de ces «hommes de métier» qui ont déjà acquis une compétence et une expérience dans leur domaine ${ }^{50}$. Au cours de l'année de formation pédagogique prévue par le règlement no. 4 du ministère de l'Éducation, les programmes universitaires destinés à la formation de cette clientele mettront un accent sur les attitudes à développer avec les élèves et sur l'acquisition d'une meilleure connaissance du fonctionnement de l'ecole et de la dynamique des groupes 5 .

Enfin, en ce qui concerne les diplômes, la CEQ reclame à nouveau la suppression du brevet «B», étant donné que la formation initiale est standardisée pour tous les futurs enseignants qui doivent maintenant se prémunir du diplôme du premier cycle d'etudes universitaires ou l'équivalent. Elle demande au ministère de l'Éducation d'accorder de justes équivalences aux détenteurs du

\footnotetext{
47. Ibid, 11-12.

48. Ibid, 2.

49. Ibid, 8-9.

50. Ibid, 13.

51. Ibid, 15 .
} 
brevet «A», de manière à ce qu'ils ne soient pas déqualifiés par les nouvelles exigences et les nouveaux diplômes.

\section{L'entrée dans le métier}

Dès la publication du Manifeste, en 1968, et pendant un peu plus de dix ans par la suite, l'un des aspects importants de la formation initiale qui retiendra l'attention de la CEQ sera le problème de la probation du personnel enseignant. On se souviendra que la CIC avait recommandé, en 1962, dans son mémoire à la Commission Parent, l'etablissement d'un tel système afin, entre autres, que les nouvelles recrues dans l'enseignement ne soient plus laissées à elles-même. Six ans plus tard (1968), la Corporation considere que les dispositifs d'encadrement et d'evaluation des candidats en période de probation demeurent inefficaces. L'amélioration de cette étape du processus au cours duquel le candidat doit faire la preuve de sa compétence à enseigner passe par l'instauration dans le milieu scolaire «des mécanismes d'évaluation et de réflexion» qui favorisent les contacts humains entre les parties concernées et entre le candidat et ses collègues. LaCEQ pose ici les principaux élements du modele qu'elle proposera, quelques années plus tard, pour remplacer le système de probation mis en vigueur par le ministere de l'Éducation ${ }^{52}$.

Au cours des deux annees suivantes, la Corporation, de concert avec le MEQ, cherchera à définir un système adéquat de probation. Cependant, à la même époque, les rapports avec l'État vont aller en se dégradant. Déjà en 1967 l'adoption du Bill 25 avait grandement mécontenté les enseignants, entre autres choses, parce qu'il instaurait un régime de négociation provinciale contre lequel s'était prononcé le Congrès de 1965. Par la suite, la longue et difficile négociation de 1968-1969 va se solder par une entente insatisfaisante pour les enseignants. Ces derniers n'apprécient guère l'attitude et le discours du gouvernement à leur endroit durant et après les événements d'octobre 1970. A l'epoque, le ministre de l'Éducation est allé même jusqu'à désigner un commissaire-enquêteur chargé de dépister les enseignantes subversifs. De telle sorte que, au cours de l'année 1971 , les instances officielles de la CEQ vont suggérer aux enseignants de refuser de participer à toute forme d'évaluation de leurs confrères. Une telle décision met entre les mains du ministre de l'Éducation l'entière responsabilité en matière de certification et de probation des enseignants ${ }^{53}$. Cette abstention fait partie d'une politique plus générale de non-participation qui aura cours entre 1971 et $1976^{54}$. Ces annees sont également marquées par des tiraillements et des luttes

52. Ibid, 30-33.

53. CEQ, op. cit, 1979, 3 .

54. Louise Clermont-Laliberté, op. cit, 1981, 42. 
idéologiques entre les fractions dirigeantes au sein de la CEQ elle-même, et par des contradictions entre la politique officielle adoptée par la Corporation et les pratiques de certains syndicats affiliés qui ne se sont pas ralliés à cette politique de non-participation ${ }^{55}$.

En 1973, la CEQ élabore et défend une nouvelle formule de remplacement du système de probation: des stages réaménagés et intégrés aux programmes de fonnation initiale. Ces stages auront une duree statutaire pour chaque secteur de spécialisation et chaque ordre d'enseignement. Ils favoriseront la collaboration entre l'institution responsable de la formation et le maître-associé de manière à mieux assurer la complémentarité entre la formation theorique et la formation pratique $^{56}$. Enseignant qualifié et expérimenté, le maitre-associé guidera le futur maître dans son apprentissage de la profession ${ }^{57}$ et travaillera de concert avec le conseiller de stage de l'université. Le maître-associé sera rémunéré pour ce travail. Le choix des maitres associés se fera par un comité de selection et sur la base de critères bien établis. Quant au conseiller de stage, il accomplira sa tâche en étroite collaboration avec le maître-associé et assumera la supervision d'un maximum de quinze stagiaires a la fois. Si on y donnait suite, cette proposition de stages réaménagés rendrait les universités plus conscientes de l'ampleur de leur responsabilite dans ce domaine.

La CEQ demeure cependant critique face a l'organisation de la formation des maîtres à cette époque. Elle reproche notamment au ministère de l'Éducation d'exercer «un contrôle de surface sur la formation» ${ }^{58}$ et de manquer «d'une politique coherente» dans ce secteur. Le Conseil des universités tente depuis 1974 «d'amener des correctifs, de coordonner et de planifier dans la province». Mais cet organisme n'a qu'un pouvoir consultatif. Au bout du compte, les institutions universitaires agissent indépendamment les unes des autres et en labsence de toute consultation des intéressés eux-mêmes 59 .

En 1976, la politique de non-participation des enseignants au comité de probation est révisée. Le Bureau national ef le Conseil géneral de la Centrale d'enseignement votent une série de propositions invitant les syndicats affiliés à sieger de nouveau au comité local et provincial de probation et demandent au représentant des enseignants d'aller y défendre les intérêts đu probaniste. En décembre 1979, la Centrale publie un nouveau document consacré à la probation dans lequel elle dénonce le pouvoir discrétionnaire dont use le ministre de l'Éducation, en vertu du règlement $n^{\circ} 4$, pour modifier chaque année les règles relatives à l'autorisation légale d'enseigner. Les règles édictées pour 1979-1980

55. CEQ, op. cit, 1979, 4.

56. CEQ, L'organisation de la formation des maîtres, Approche théorique-approche pratique (Québec, CEQ, octobre 1973), 5.

57. Ibid, voir le détail des tấches, 28 .

58. Ibid, 41 .

59. Ibid, 15 . 
ont pour effet d'augmenter le pouvoir du directeur en lui accordant «le droit de vie ou de mort»sur le probaniste ${ }^{60}$. $\AA$ toutes fins pratiques, les deux ou trois années de probation se retrouvent sous l'entière responsabilité du directeur d'école (ou de son représentant). C'est d'ailleurs le directeur qui choisit l'enseignant breveté faisant partie du comité de probation et c'est lui qui demeure également «le seul signataire officiel du rapport de probation et de la formule de recommandation" ${ }^{61}$.

En insistant de la sorte pour déplacer le centre de décision du lieu de travail vers les universités, la CEQ cherche ainsi à diminuer l'emprise qu'exercent sur ses nouveaux membres les administrateurs scolaires et le ministère de l'Éducation. Bien plus, elle vise à élargir le contrôle des enseignants sur la question de la permanence en tentant d'inscrire dans les conventions collectives locales et provinciales les modalités d'acquisition de ce statut. Elle propose à cette fin aux commissions scolaires et au ministère de l'Éducation de s'inspirer de ce qui est prévu sur cette question dans les conventions collectives des enseignants des collèges d'enseignement général et professionnel (CEGEP) ${ }^{62}$.

\section{Une direction tri-partite du procès de formation}

Pour mettre en pratique les propositions qu'elle avance, la CEQ croit qu'il faudrait considerer les facultés des sciences de l'éducation comme des facultés professionnelles et leur réserver un traitement en conséquence. Les sommes versées par l'État aux universités pour couvrir les dépenses imputables aux clientèles qui fréquentent de telles facultés doivent donc faire l'objet de subventions autonomes et non-transférables à d'autres unités. Ces facultés profession. nelles assumeraient l'organisation et l'administration de tous les programmes de formation et de perfectionnement du personnel enseignant et conduiraient des recherches dans les domaines qui relevent de leur champ de compétence. Les syndicats d'enseignants participeraient à la gestion de ces facultés. Les universites ne devraient pas avoir seules le contrôle des institutions de formation $^{63}$. Ici comme ailleurs, la Centrale veut demeurer Ie chien de garde des interêts de ses membres. Dans la relation triangulaire qui la lie au ministère de l'Éducation et aux universités, elle se donne le rôle d'intermédiaire, d'arbitre ou «de juge soucieux du maintien de la qualité de la formation du personnel enseignant». Elle se voit comme etant «l'axe d'équilibre» et «le pivot du

60. CEQ, op. cit, 1979, 14.

61. Ibid.

62. Ibid, 16.

63. CEQ, op. cit, mars, 1980, 19. 
balancier» ${ }^{64}$. Cette solution, qui revient en fait à une sorte de gestion tri-partite du procès de la qualification, passe par la création d'un conseil à l'interrieur de chaque université et d'un conseil national de la formation et du perfectionnement des enseignants qui serait le maitre d'œuvre national de la formation et du perfectionnement des enseignants. En plus d'assumer la coordination des universites dans le domaine, cet organisme national-composé à parts égales des representants des universités, des associations d'enseignants et du gouvernement-aurait la responsabilité de l'élaboration «des politiques de formation et de perfectionnement universitaires des enseignants, de la détermination des besoins, des orientations, des objectifs et des priorités, de la détermination des critères, de l'approbation des programmes approuvés aux fins de la certification» 65 .

Le conseil national dont la CEQ recommande la création ressemble bien à cet autre organisme qui sera conçu, cinq plus tard, par un haut fonctionnaire du ministère de l'Éducation dans un énoncé de politique en matière de formation et de perfectionnement des enseignants qui ne verra pas le jour, en raison, notamment, du changement du parti politique au pouvoir ${ }^{66}$. Si elle devait un jour se concrétiser, la Commission de la formation et de la titularisation en enseignement, dont la création est prévue par le projet d'énoncé de politique, serait composée, dans sa grande majorité, d'enseignants-neuf sur dix-sept-et comprendrait des représentants des parents, des commissions scolaires, du ministère de l'Éducation et des universités. Contrairement au conseil national suggéré par la $C E Q$, la commission n'aurait pas la responsabilite de coordonner l'action des universités dans le domaine de la formation et du perfectionnement des enseignants, ne serait pas autonome mais rattachée au Conseil superieur de l'Éducation, et aurait, enfin, un pouvoir consultatif plutôt que décisionnel ${ }^{67}$.

\section{CONSERVER LES ACQUIS, RÉORIENTER L'IDÉOLOGIE ET L'AC'TION!}

En plus de continuer de suivre de près toute la question scolaire-nécessité d'élaboration d'une politique de la formation et du perfectionnement des enseignants, de remplacement de la période de probation par un stage intégré au programme de la formation initiale, d'amelioration des stage en milieu scolaire,

64. Ibid, 4 .

65. Ibid, 26.

66. Ministère de I'Éducation du Québec (MEQ), Enseigner au Québec. Formation el titularisation. Énoncé de politique (projet de) (Québec, ministère de l'Éducation, 1985).

67. Ibid, 125-126. 
etc $^{68}$ - la , laQ va s'intéresser à plusieurs autres questions, telles la question nationale, les droits et libertes démocratiques, les droits de l'enfant, la lutte des femmes, les droits syndicaux, la paix mondiale. Ces années sont celles d'une récession économique qui s'accompagne d'une montee du conservatisme, ici comme dans beaucoup d'autres pays. L'État revient sur certains acquis de la reforme des annees 1960 et le syndicat tente d'en minimiser l'impact sur ses membres et sur sa propre action. Mais la conjoncture économique et politique pèsera de tout son poids sur les orientations et l'action de la Centrale qui, pour éviter l'éclatement, accepte de faire un compromis idéologique qui satisfait les factions qui la composent: renoncer au discours radical et contre-hégémonique tout en continuant de militer pour une ecole démocratique et une société juste et équitable pour les plus démunis.

\section{De la pédagogie de conscientisation au pluralisme des modèles pédagogiques}

Contrairement à ce que prétendent certains ${ }^{69}$, la CEQ ne s'est jamais désintéressée de la pédagogie en général, mais plutôt d'une certaine pédagogie, celle qui conçoit l'enseignant comme un agent dont le rôle consiste à inculquer aux enfants de toutes les classes sociales les valeurs et les idéologies qui assurent la légitimité et la permanence de l'ordre social établi. On le sait, c'est durant la première moitié des annees 1970 - et surtout en réaction à l'action législative répressive menée par l'État à la fin des années soixante-que la CEQ développe, et tente de diffuser auprès de ces membres, une pédagogie critique qui définit la société comme étant divisée en classes dont l'une exploite les autres et l'enseignant, comme un agent socialement engagé dont le rôle est de dévoiler, en vue de les combattre, l'exploitation et les idéologies qui la fondent. Cette pédagogie de conscientisation à laquelle les enseignants sont tous conviés fait

68. En 1983, le ministère de l'éducation du Québec (MEQ) diffuse pour consultation auprès des organismes scolaires un document portant sur La formation et le perfectionnement des enseignants du primaire et du secondaire, vers des aménagements, document de consultation (Québec, ministère de l'éducation, juin 1983), 154 p., pagination multiple. C'est en partie dans sa réaction à ce document que la CEQ réaffirme sa position sur les questions évoquées. Voir CEQ, Position de la Centrale de l'enseignement du Québec sur les fiches de discussion portant sur la formation et le perfectionnement des enseignants du primaire et du secondaire (Québec, CEQ, juin 1984).

69. Voir à ce sujet, par exemple, Claude Lessard, «Statut des enseignants: quelques éléments d'analyse et facteurs d'évolution", dans Fernand Dumont, dir., L'Education 25 ans plus tard! Et après? (Québec, IQRC, 1990), 339-340; voir aussi Jean-Paul Desbiens, «Syndicalisme et qualité de l'enseignement», L'école coopérative 44 (juin 1978), 7-11. 
partie de l'offensive idéologique contre-hégémonique que la CEQ, et d'autres centrales syndicales, entreprend pour s'opposer à l'idéologie dominante que véhiculent les programmes, les manuels scolaires et les activités conçus par les agents idéologiques de l'État ${ }^{70}$.

L'élection d'un parti politique, en principe, favorable à la cause des enseignants-le Parti Quebécois (1976) - la division que cette élection va nourrir au sein des équipes syndicales dirigeantes et le contexte de chute démographique et de récession économique que connaîtra le Québec dès la fin des années 1970 vont pousser la CEQ vers une reformulation plus moderée de son discours sur l'école et la pedagogie. En un mot, la CEQ laisse tout simplement tomber le discours critique radical et contre-hégémonique, auquel elle conviait tous les enseignants de la première moitié de la décennie 1970, pour s'engager dans la voie du pluralisme idéologique consacrée par le Rapport Parent et chère aux intellectuels québécois, les universitaires et les professeurs des facultés des sciences de l'éducation surtout. C'est précisément entre 1980 et 1982 que s'accomplit ce virage idéologique qui est le résultat d'un compromis entre «deux courants de pensée d'égale force à l'intérieur de la centrale: l'un veut un changement fondamental du système économique, l'autre plaide pour des réformes sans remettre en question le syst ème capitaliste». Dès juin 1982, selon Jacques Rouillard, la nouvelle orientation de la CEQ est fixée: «ll n'est plus question d'abolition du système capitaliste, de luttes de classe ni d'opposition irréductible entre l'État et les travailleurs ${ }^{71}$. La centrale continue cependant de soutenir que les interêts des enseignants demeurent liés à ceux des autres travailleurs.

Ce dualisme idéologique, on le retrouve au coeur même du discours de la CEQ sur la formation et le rôle des enseignants comme on peut le déceler dans sa réaction au rapport de la commission d'étude sur les universités québécoises (commission Angers), instituee en 1977 par le gouvemement. La commission confie l'analyse de la situation de la formation des enseignants au sein de ces établissements à un comité dont les travaux vont constituer, durant près de deux ans, un moment important ou la CEQ - comme beaucoup d'autres organismes, d'ailleurs - affirmera la conception qu'elle se fait de la preparation du personnel enseignant, le sens qu'elle donne à cette préparation et le rôle qu'elle entend y jouer. C'est, entre autres, dans ses commentaires sur le rapport du Comité d'étude sur la formation et le perfectionnement des enseignants (CFPE), publié en mai 1979, que la CEQ exprime sa position. Le rapport du CFPE recommande que le «respect du pluralisme des conceptions de l'éducation, des conceptions de l'homme et des conceptions de la société soit reconnu comme condition préalable à tout discours démocratique sur les orientations philosophiques devant présider

70. Marta Anadon, L'école québécoise: jeux et enjeux de forces sociales, 1970-1980, op. cil, $1989,188-189$.

71. Jacques Rouillard, Histoire du syndicalisme au Québec, des origines à nos jours, op. cit, 370 . 
à la formation et au perfectionnement des enseignants» et que chaque institution jouant un rôle dans la formation et le perfectionnement du personnel enseignant s'assure de la coexistence, dans ses programmes comme dans les pratiques pédagogiques de ses professeurs, d'une pluralité de philosophies, de valeurs et de modeles d'éducation ${ }^{72}$. La CEQ adhère à cette analyse et presse le ministère de l'Éducation à opérer les changements qui s'imposent pour concrétiser l'idéal qui la sous-tend. Aussi, les programmes et les activités de formation ne s'aligneront plus sur un seul modèle pédagogique, ni ne s'accommoderont d'une conception monolithique de l'éducation et de la societe $e^{73}$.

Ainsi, après avoir réclamé, sans beaucoup de succès une dizaine d'années durant, la légitimité de son modèle pédagogique--pédagogie de conscientisation-et incitéses membres a faire obstacle aux modèles proposés par le ministère de l'Éducation, la CEQ change maintenant de perspective et d'argumentation: la société est pluraliste, l'école doit refléter le pluralisme social et idéologique et c'est à l'enseignant que revient le choix de l'approche pédagogique. S'il ne veut pas être réduit au rôle d'un outil de transmission de l'idéologie dominante, l'enseignant doit cependant être en mesure de comprendre et de remettre en cause le modele officiel d'école, d'éducation et de société ${ }^{74}$. Dans cette optique, l'université représente l'endroit privilégié où il peut acquérir les concepts et expérimenter les approches qui le préparent à la pratique de cette démarche critique. Selon la conception que se fait d'elle la CEQ, «la démocratisation scolaire exige des enseignants qu'ils aient un rôle instrumental dans la prise de conscience par les travailleurs des valeurs, idéologies, et objectifs véhiculés par le modèle» dominant d'éducation et de société ${ }^{75}$. C'est pour cette raison d'ailleurs qu'elle se déclare favorable à l'insertion dans les programmes de formation et de perfectionnement des enseignants d'une initiation «aux caractéristiques socio-économiques du milieu et aux implications pédagogiques qui en découlent» ${ }^{76}$. En plus de resserrer les liens entre la theorie et la pratique, les programmes de formation initiale devront intégrer la période de probation à laquelle sont soumis, depuis la réforme des années 1960, les nouveaux enseignants ${ }^{77}$. Concrètement, cela signifie le prolongement des stages, un meilleur

72. Conseil des universités, Comité d'étude sur la formation et le perfectionnement des enseignants, Rappon, (Québec, Gouvernement du Québec, mai 1979), 18-19.

73. CEQ, Projet de prise de position de la CEQ sur le rapport du C.F.P.E. (C.E.U.) (Québec, CEQ, mars 1980), 8.

74. Ibid, 9.

75. Ibid.

76. Ibid, 10. Cette recommandation a été avancée par le Comité d'étude sur la formation et le perfectionnement des enseignants. Voir Conseil des universités, Comité d'étude sur la formation et le perfectionnement des enseignants, Rapport, op. cit, 30.

77. CEQ, op. cit, mars 1980, 1. 
encadrement, une définition précise du rôle des intervenants et l'elimination de l'actuel système de probation.

Enfin, alors qu'elle réclamait, en 1962, dans son mémoire à la commission Parent, pour couronner les études pédagogiques, l'octroi d'un brevet standard temporaire qui, une fois passé avec succès le stage de probation, débouche sur l'obtention d'un permis permanent d'enseigner, en 1980, la CEQ suggère que le permis permanent soit délivré dès la sortie de l'universite $\hat{j}^{78}$. Il s'agit là, pour elle, du seul moyen d'éviter la déqualification de la formation acquise. En modifiant constamment les règles de certification, le ministère de l'Éducation condamne les enseignants à un interminable recyclage ${ }^{79}$, ce qui est loin d'être le cas des autres professionnels ${ }^{80}$. II s'agit pour, la Centrale syndicale, d'une idéologie et de stratégies employées par le MEQ pour masquer «l'absence d'une politique de main-d'oeuvre dans l'enseignement» ${ }^{81}$. L'enseignement constitue une spécialisation en soi. Celui qui enseigne est spécialisé en enseignement. Les disciplines de specialisation ou les secteurs d'affectation ne devront donc pas être mentionnés dans le libellé des diplômes.

\section{Toujours pour une école de masse}

Le Congrès de juin 1980 adopte une plate-forme globale qui oriente le discours et l'action syndicale. Il s'agit d'un document qui definit la conception que se fait la CEQ de l'école primaire et secondaire et du rôle que doivent y jouer les enseignants. Dans ce document intitulé Proposition d'école, la CEQ préconise une école commune et un système scolaire et des structures non confessionnels $^{82}$. L'école est une institution destinee à répondre aux besoins de la masse d'enfants qui la fréquentent, enfants issus, pour une bonne part, des milieux ouvriers et des classes populaires. Sa fonction doit se conformer à la «conception evolutive», que se fait la CEQ de la personne, conception «qui reconnait la prédominance de l'acquis sur l'inné». C'est pour cette raison qu'il faut bannir toute utilisation de tests visant à mesurer l'intelligence et les aptitudes, tests qui ont inevitablement pour effet de classer les enfants dans des voies de garage ou tout simplement de les éliminer du circuit scolaire.

Si elle veut être au service de la majorité des enfants qui la fréquentent, l'école doit véhiculer des valeurs liées au vécu et aux interêts des travailleurs: la

\section{Ibid, 10.}

79. Ibid, 13.

80. La CEQ écrit à ce sujet: «Connâtiton beaucoup d'avocats, de notaires ou d'architectes qui, une fois sur le marché du travail, voient leur formation remise en question?», Ibid, 14.

81. Ibid.

82. CEQ, Proposition d'école (Quélxec, CEQ, juin 1980), 9. 
solidarité, l'entraide et l'esprit d'équipe, la promotion collective (sans négliger le dépassement de soi), l'action et le changement, le travail bien fait. Cette école emploiera une pédagogie de masse et des pratiques scolaires visant le progrès de tous et non la formation d'une élite. Cette pédagogie évitera la division des élèves en forts et faibles selon leurs résultats scolaires, adaptera le processus et les activités d'apprentissage aux réalites concrètes de l'enfant et cherchera les causes de son échec ou de son retard scolaires non dans les traits de sa personnalité, mais dans les conditions d'existence de sa famille et dans les pratiques scolaires auxquelles il est exposé. Cette pédagogie de masse s'appuie sur la conviction, découlant elle-même de la conception évolutive de la personne évoquée plus haut, qu'il existe un potentiel de transformation chez tous les individus et que ce sont les conditions d'existence et les pratiques scolaires qui rendent possible ou entravent l'éclosion d'un tel potentiel ${ }^{83}$.

L'école secondaire continue d'être un espace où se pratique la discrimination a l'endroit des enfants des travailleurs. La formation de base qui y est dispensée ne répond pas aux besoins des élèves, dont $73 \%$ entrent dans la vie active da la fin de ce cycle. C'est pourquoi la CEQ préconise une formation commune à tous les élèves jusqu'à la cinquiène année du secondaire. Les programmes conçus à cette fin et les enseignants qui les mettent en pratique devront dévclopper chez ces futurs travailleurs la capacite de comprendre en vue de les transformer les réalités multiples auxquelles ils sont et demeureront confrontes: réalité économique, politique, culturelle, morale ou religieuse, environnementale, etc. ${ }^{84}$. La culture scolaire deviendra un prolongement et non une négation de la culture de cette classe de travailleurs. Ceux-ci y développeront donc le sens de la solidarité sociale et une conscience de leur force collective sur laquelle ils doivent $s$ 'appuyer dans la promotion de leurs interêts ${ }^{85}$. Ici comme ailleurs, la langue et les mathématiques constituent les outils essentiels de la connaissance. Si on ne veut pas qu'ils demeurent des abstractions sans grande signification pour les élèves, leur apprentissage ne doit pas être coupé de la réalité des enfants, ni utilisé à des fins de sélection et d'élimination. Enfin, l'évaluation des apprentissages doit s'insérer dans un processus de correction et non se transformer en un mécanisme d'élimination. Elle développera l'entraide et la solidarité plutôt que l'émulation. Une évaluation qui a pour but de sélectionner et d'éliminer entrave, du même coup, l'accès au savoir de base à une partie importante des étudiants. Et cela va à l'encontre des objectifs d'une école commune et démocratique.

83. Ibid, 11-12.

84. Ibid, 14.

85. Ibid, 14-15. 


\section{Les facultés des sciences de l'éducation: un bilan et ses lacunes}

En 1987, le Conseil des universites termine une étude faisant un bilan de l'évolution des facultés des sciences de l'éducation. Comme dans toutes les occasions du genre, les organismes et les groupes concernés ont exprimé leur point de vue sur le contenu du bilan ${ }^{86}$. En janvier 1987 , le comité exécutif de la Commission des enseignantes et enseignants des commissions scolaires, instance de la CEQ, commente ce document. Il constate, entre autres, que l'élude du Conseil des universités a le mérite de mettre en évidence «la variabilité étonnante des types de programmes de formation et de leurs structures» ${ }^{87}$, démontrant par-là l'urgence de procéder à une «certaine uniformisation» et la nécessité d'evaluation de ces programmes. Quant aux contenus de ces programmes de formation initiale, le comité exécutif rappelle l'évaluation asse $z$ sévère qu'en ont faite les enseignants, et dont le Conseil des universités ne tient pas compte dans le bilan qu'il dresse. Les recherches menées entre 1978 et 1985 par le ministère de l'Éducation ${ }^{88}$, de même que les nombreuses interventions faites dans le cadre des États généraux de l'Éducation tenus en 1986, ont bien mis en évidence l'evaluation plutôt négative que font les enseignants de leur formation initiale dans bon nombre de domaines: l'aide aux enfants en difficulté, la solution aux problèmes de discipline, l'adaptation de l'enseignement aux caracteristiques socio-économiques des élèves, la compréhension des changements introduits dans les écoles, les relations avec les parents, les connaissances relatives aux aspects sociaux, politiques et économiques de l'éducation ainsi qu'aux aspects administratifs du systeme scolaire. Il s'agit là de lacunes importantes dans la préparation au métier ${ }^{89}$. Les enseignants préconisent une formation initiale qui développe chez eux les habiletés en animation, en travail d'équipe, en communication, en création de matériel didactique et pédagogique, en somme une formation qui prépare plus adéquatement aux relations inter-personnelles et

86. Ce bilan a donné lieu à l'élaboration d'un Avis au ministre de l'Enseignement supérieur et de la Science. Voir Conseil des universités, Le développement du secteur de l'éducation. Avis du Conseil des universités au ministre de l' Enseignement supérieur et de la Science (Ste-Foy, Conseil des universités, 1988).

87. Ibid, 2 ,

88. Il s'agit d'une recherche sur les enseignants et les enseignantes du Québec, dirigée par Roger Cormier, Claude Lessard et collaborateurs, qui a donné lieu à la publication de 9 volumes entre 1979 et 1985 . Voir surtout le volume 5: Roger Cormier, Claude Lessard, Louis Toupin et Paul Valois, Les enseignantes et les enscignants du Québec, une étude socio-pédagogique: la formation et le perfectionnement (Québec, Gouvernement du Québec, 1980).

89. CEQ, Le Bilan du secteur de l'éducation présenté par le Conseil des universités: réactions et commentaires (Québec, CEQ, janvier 1987), 2. 
permet une meilleure connaissance des programmes à enseigner ainsi qu'une certaine polyvalence ${ }^{90}$.

Le comité exécutif reproche ensuite au Conseil des universités son silence sur la très importante question des stages et de la probation. Le bilan demeure donc incomplet et lacunaire. De plus, le Conseil des université n'a pas modifié ses positions sur les règles d'affectation et sur le système de perfectionnement. Il oublie qu'il n'a pas à «remettre en question» ces élements qui sont des objets de convention collective et qui, de ce fait, appartiennent au champ de la négociation. D'une manière à peine voilé, la CEQ invite le Conseil des universités à ne pas aborder des questions comme celles-là, qui ne relèvent pas de son champ de compétence ${ }^{91}$. Enfin, le comité exécutif s'attarde assez longuement sur la nature de la recherche en éducation et considère qu'il est temps que les chercheurs universitaires se mettent enfin à l'écoute du milieu scolaire.

Bref, au cours des années 1980, la CEQ continue de définir Ia mission sociale de l'école comme étant de contribuer à la formation de citoyens et de citoyennes responsables, autonomes, sociables et possédant un esprit critique ${ }^{92}$. Pour ce faire, une telle formation doit avoir un contenu qui colle davantage au vécu de l'élève, une pédagogie de masse, une évaluation de type correctif, une diminution du nombre d'élèves par classe, une amélioration des conditions de vie des familles, des recherches et des pratiques visant à rendre les savoirs scolaires plus accessibles aux élèves ${ }^{93}$. Cette formation fondamentale doit également faire place à la culture technique qui développera chez les enfants l'esprit d'initiative et le potentiel innovateur plutôt que la faculté d'adaptation et l'instinct de dépendance ${ }^{94}$. Cette formation de base large et polyvalente est rendue nécessaire, notamment par «la restructuration du marché du travail et l'innovation technologique» ${ }^{95}$. Un tel projet d'école exige «un changement d'attitudes sociales et pédagogiques chez plusieurs travailleurs de l'enseignement». Et c'est précisément à l'acquisition de telles attitudes que devront contribuer les activités et les programmes de formation du personnel enseignant.

\section{CONCLUSION}

Saisie de l'intérieur, l'évolution du discours syndical reflète bien les préoccupations majeures de la principale organisation professionnelle des enseig-

90. Ibid, 3.

91. Ibid, 8 .

92. $\mathrm{CEQ}$, Pour alimenter notre participation aux Érats généraux sur la qualité de l'Éducation (Québec, CEQ, mars 1986), 11.

93. Ibid, 13.

94. Ibid, 7.

95. Ibid. 
nants du Québec durant les soixante demières années. Le discours et l'idéologie ont changé. Les moyens d'action aussi. Mais les questions fondamentales pour Iesquelles luttent les enseignants depuis toujours ne se sont pas beaucoup modifiées. On peut même dire qu'elles peuvent être ramenées à une seule dont decoule tout le reste: la recherche d'une reconnaissance sociale à la mesure de ce qu'ils croient être l'importance de leur mission éducative et sociale. Évidemment les termes et les modalités de cette demande de reconnaissance varient avec le temps, en fonction des classes détentrices des pouvoirs politiques, économiques et idéologiques, et selon les rapports que les enseignants entretiennent avec ces classes.

De la constitution de la Corporation générale des instituteurs et institutrices catholiques du Québec (CIC) en 1945 jusqu'au milieu des années 1960, dans un Québec encore largement dominé, sur le plan idéologique du moins, par les élites cléricales, les modalités de la reconnaissance de l'enseignant, de son statut social et de son rôle, sont définies par référence à la doctrine sociale de l'Église catholique telle que posee par les trois principales encycliques papales ${ }^{96}$ de 1891 , 1931 et 1937: regroupement dans une association de type professionnel, adhésion à la religion de la majorité et participation active d̀ sa retransmission aux jeunes générations par le biais de l'école, bannissement des idéologies, des doctrines, des méthodes et des procédés pédagogiques non conformes au caractère confessionnel des écoles catholiques. Ici, la valorisation đu statut et du rôle passe par l'élevation du degré de qualification, par le contrôle des criteres d'accès à la profession ef par la participation des enseignants aux instances décisionnelles locales, régionales et centrales. Pas de contestation cependant de type syndical, ni de confrontation ouverte avec l'employeur ou de remise en cause des fondements de l'ordre établi. Telles sont les conditions de la caution cléricale à l'action des enseignants et les limites a l'intérieur desquelles cellemci devait être exercée. L'Église assura les bases idéologiques du mouvement syndical, l'État, les cadres juridiques restreints dans lesquels il dut évoluer cahin-caha au cours des années 1930-1960.

Les amées 1970 voient un déplacement de l'idéologie et de l'action syndicale vers un radicalisme jusque-là insoupçonné dont la conjugaison d'un ensemble d'éléments conjoncturels permet d'en saisir la signification: luttes pour la liberation des pays colonises, tombee de la censure contre les idéologies radicales, marxistes surtout, triomphe relatif des doctrines néo-liberales, retrait de l'Église des affaires temporelles et laïcisation des institutions sociales et des structures syndicales, intervention de plus en plus grande de l'État dans les relations de travail - dans le champ de la négociation, par exemple- confrontation de plus en plus ouverte entre les centrales syndicales et l'État ${ }^{37}$. Sur le

96. Rerum novarum (1891), Quadragesimo anno (1931) et Divini Redemptoris (1937).

97. Voir à ce sujet $M$ 'hammed Mellouki, «La qualification des enseignants, un enjeu et 
plan strictement scolaire, la fin des années soixante est marquee, on ne le soulignera jamais assez, par un vide idéologique qui a aussi contribué à la réorientation du discours et de l'action de la Centrale de l'enseignement du Québec. Les anciens programmes et manuels scolaires étaient déjà rangés au musee de l'histoire et avec eux les doctrines et les orientations normatives qui ont façonné jusque-là les finalités de l'école et déterminé le rôle des agents scolaires. Les témoins de l'époque s'accordent pour dire que la réforme de l'education s'est davantage concentrée sur la refonte des structures scolaires et des cadres juridiques que sur la philosophie de l'éducation et la mission de l'école dans l'ordre social qui prend forme ${ }^{98}$. Dans un contexte de changement comme celui-ci, les groupes sociaux cherchent, entre autres par le moyen de leurs associations professionnelles, à renforcer leur emprise sur les institutions éducatives, sur la définition de leurs objectifs, sur la prise de décision, sur les pratiques pédagogiques. Ce fut le cas de la CEQ entre 1967 et 1976 surtout.

C'est à la lumiere de ces données contextuelles et de la crise grave qui affecte les relations entre l'État et le syndicat des enseignants qu'il faut donc comprendre le virage idéologique que prend la CEQ au début des années 1970 et le sens des revendications qu'elle défendra relativement à la valorisation de l'enseignant et de son statut. Dans la société sans classes sociales qu'elle prône, la Centrale réserve à l'enseignant un rôle d'éveilleur de conscience et de défenseur des valeurs d'égalité, d'équité et de justice sociale. Elle fait de lui le pivot et le moteur du changement social, le pilier de l'ordre social nouveau. C'est donc au prix d'une réhabilitation du rôle fondamentalement idéologique de l'enseignant, et uniquement à ce prix, que la CEQ réussit à mettre en évidence toute l'importance du personnage pour la société. Quant à savoir si elle a résussi à convaincre la société, l'État et les enseignants eux-même, c'est loin d'être sûr à en croire la rumeur.

La fin des années 1970 ouvre la voie au compromis dans l'idéologie et l'action syndicales qui s'achevera au cours de la décennie suivante. L'élection en 1976 du Parti Québécois a la tête de l'État provincial pose à la CEQ le problème de la nature et du degré de son engagement dans l'action socio-politique, contre-offensive qu'elle a maintenue depuis le début des années 1970. Puisqu'il $s$ 'agit d'un parti en principe favorable à la cause des enseignants, qui compte un bon nombre d'entre eux dans ses rangs et qui a été porté au pouvoir grâce à leur

ses acteurs», loc. cit, 1991a.

98. Réginald Grégoire, L'évolution des politiques relatives aux programmes d'études du primaire et du secondaire calholique francophone du Québec (Québec, ENAP, 1987), 197; Roland Vinette, «De l'école normale à l'université», dans J.\}. Jolois et R. Piquette, La formation des maitres et la révolution tranquille (Montréal, Université du Québec à Montréal, 1988), 60-80; Roland Vinette, «es structures péáagogiques», dans En collaboration, Le rapport Parent, dix ans après (Montréal, Bellamin, 1975), 59-73. 
appui, la question qui se pose à la Centrale est la suivante: est-il justifié de continuer d'identifier l'état québécois à la classe dominante et de mener contre lui une lutte radicale et systématique? La cœxistence des ailes radicale et péquiste au sein de la CEQ est soumise à dure épreuve et les orientations idéologiques de la Centrale sont remises en cause dès le congrès de 1978. Yvon Charbonneau, représentant de la faction radicale, est défait lors de ce congrès. Robert Gaulin, porte-parole de l'aile péquiste et partisan d'une action syndicale plus modéree, est élu président de la CEQ. «ll plaide, écrit Jacques Rouillard, pour un plus grand pluralisme à la direction de la Centrale et pour un rapprochement entre la direction et ses membress ${ }^{99}$.

La suite est connue. En 1980, le congrès général de la CEQ adopte un document d'orientation qui, même s'il ne met pas carrement fin au radicalisme de son idéologie socio-politique, engage néanmoins la Centrale dans la voie du pluralisme idéologique que confirment, on l'a vu, d'autres documents exprimant la position de l'organisme sur les problèmes scolaires et sociaux qui se posent durant la décennie. La CEQ continuera de se dire en faveur d'une société égalitaire et démocratique, mais plus question d'opposition farouche au systeme capitaliste, ni de son abolition comme condition a la realisation du projet de société défendu dans les documents publies entre 1972 et 1975. Le terme de «classe sociale» lui-même est à toutes fins pratiques absent des résolutions adoptées lors des congrès géneraux entre 1980 et 1990, même si la présidence de la CEQ fut à nouveau occupée par Yvon Charbonneau et même si le Parti Québécois n'est plus à la tête du gouvernement. Aux concepts jadis utilisés de «classe ouvrière», de «classe exploitée» ou de «prolétariat» on préfère aujourd'hui des termes, de connotation idéologique plus anodine, de «milieux socio-économiquement faibles» ou de «milieux défavorisés». La pédagogie de conscientisation continue d'être le modele defendu par la $\mathrm{CEQ}$, mais elle ne constitue dorenavant qu'une approche parmi d'autres que les enseignants sont appeles à suivre. La demande de valorisation des enseignants, de leur statut et de leur rôle est à son degré zéro.

On le voit donc, au cours de la période explorée, le discours syndical porte sur un seul et même objet même si cet objet devient, par moment, prétexte à la formulation de projet d'école et de société: la reconnaissance sociale de l'enseignant, de son statut et de son rôle. L'hypothèse qui semble se dégager de l'analyse est que plus le contexte économique est prospère, plus les institutions sociales se läicisent, les droits syndicaux sont reconnus et les liens entre les syndicats étroits, plus la demande d'une reconnaissance sociale de l'enseignant est totale. Dans des contextes caractérisés par une récession économique, par un conservatisme idéologique et politique et par un relâchement des liens syndicaux, la demande de reconnaissance sociale est minimaliste. Elle vise à protéger le

99. Jacques Rouillard, op. cit, 1989, 367 . 
personnel enseignant contre l'augmentation de sa tâche, la dégradation de ses conditions de travail, la dequalification professionnelle, la réduction de son salaire ou la diminution de son autonomie professionnelle. En cela, le discours syndical est empreint d'un réalisme à toute épreuve. Il porte en lui la marque des differentes conjonctures économiques, politiques et sociales que le Québec a traversees au cours des soixante dernières annees. 
